\title{
Analysis of the expression of KIR and HLA-Cw in a Northeast Han population
}

\author{
YU HAN $^{1 *}$, LING ZHAO ${ }^{2 *}$, ZHENYU JIANG ${ }^{2}$ and NING MA ${ }^{2}$ \\ ${ }^{1}$ Jilin Blood Center, Changchun, Jilin 130033; ${ }^{2}$ Department of Rheumatology, \\ First Hospital, Jilin Unversity, Changchun 130021, P.R. China
}

Received June 3, 2012; Accepted October 8, 2012

DOI: $10.3892 /$ etm.2012.763

\begin{abstract}
The aim of this study was to investigate the expression of the human leukocyte antigen (HLA)- $\mathrm{Cw}$ and killer cell immunoglobulin-like receptor (KIR) genes in a Jilin Han population and to provide a theoretical basis for further studies of their roles in disease. A total of 154 unpaid Jilin Han blood donors were selected and KIR and HLA-Cw genotyping was performed using PCR-SSP. Recognition of HLA-Cw and the corresponding activatory or inhibitory KIR receptor was distinguished according to the identification of HLA-Cw and KIR. In the present study, the expression frequency of HLA-C2 $2^{\mathrm{Lys} 80}+2 \mathrm{DL} 1$ was $27.27 \%$, HLA-C $1^{\text {Asn80 }}+2$ DL2/2DL3 was $68.83 \%, 2$ DS2+HLA-C1 ${ }^{\text {Asn80 }}$ was $9.74 \%$ and $2 \mathrm{DS} 1+\mathrm{HLA}-\mathrm{C} 2^{\mathrm{Lys} 80}$ was $9.74 \%$. Of the individuals in the study, $72.08 \%$ expressed only KIR2DL1 without HLA-Cw, 21.43\% expressed only KIR2DS1 without HLA-Cw ${ }^{\text {Lys }}$-KIR2DL1 and $2.60 \%$ expressed only KIR2DS2 without HLA-Cw ${ }^{\text {Asn }}$-KIR2DL2/L3. In conclusion, the expression of inhibitory HLA-Cw-KIR is higher than the expression of activating HLA-Cw-KIR and approximately $20 \%$ of the individuals separately expressed the activated HLA-Cw-KIR in the Jilin Han population in the present study.
\end{abstract}

\section{Introduction}

Killer cell immunoglobulin-like receptor (KIR), expressed by natural killer (NK) cells and certain T cells, is a type of CD158 glucoprotein (1). Through the recognition of human leukocyte antigen (HLA)-I, KIR regulates the cytoactivity of the effector cell, begins the immunological process and regulates cytokine secretion. KIR gene clusters have extremely complex gene structures gradually formed from the single gene structure of

Correspondence to: Professor Zhenyu Jiang, Department of Rheumatology, First Hospital, Jilin University, No. 71 Xinmin Street, Changchun 130032, P.R. China

E-mail: jiangzhenyu2012@163.com

*Contributed equally

Key words: killer cell immunoglobulin-like receptor, human leukocyte antigen- $\mathrm{Cw}$, ligand, Jilin Han ethnicity
KIR3DX1 (2). The human KIR gene cluster contains fifteen genes and two silencers. The KIR gene cluster encoding the receptor contains protein guide sequences (exons 1 and 2), the extracellular domain (exons 3-5), stem (exon 6), transmembrane domain (exon 7) and intracellular domain (exons 8 and 9). The KIR gene cluster genes are named according to their extracellular domain and intracellular domain structural features. An extracellular domain with two similar immunoglobulin domains would be named 2D, or 3D if it had three similar immunoglobulin domains. KIR genes with longer intracellular domains and transduction inhibitory signals are designated L, while those with shorter intracellular domains and transduction activition signals are designated $\mathrm{S}$. The main ligand of KIR is the HLA-I class antigen. Following the recognition of various HLAs, the KIR receptor determines whether the object belongs to the 'self' or not, thus making it extremely important in immune regulation $(3,4)$. The KIRs may be divided into two categories: stimulatory KIRs (sKIRs) and inhibitory KIRs (iKIRs).

The specific receptors of certain inhibitory KIR class HLA-I molecules are already known. The ligand of the inhibitory genes KIR2DL1, -2DL2 and -2DL3 is HLA-C. Based on the amino acid combination at the heavy chain loci 80, HLA-C has been divided into two groups. In the HLA-C1 group, the amino acid of site 80 is lysine, while in the HLA-C2 group it is aspartic acid. The ligand of KIR2DL1 is HLA-C2 and the ligand of KIR2DL2/2DL3 is HLA-C1 (5). KIR2DL1+HLA-C2 combined excitatory and inhibitory signals are stronger than those of KIR2DL2/2DL3+HLA-C1 (6). The ligand of KIR3DL1/S1 is the HLA-B antigen which contains a Bw4 structure. It has been reported that KIR3DL1/S1 is able to combine with the HLA-A antigen, which also contains a Bw4 structure (7). The ligand of KIR3DL2 is HLA-A3 or HLA-A11. The ligand of KIR2DL4 is HLA-G. The ligand of KIR2DS1-5, an activating gene of KIR, may be an HLA class I antigen although there is no direct evidence to support this theory. Individuals with variations in the KIR gene cluster number and type may be divided into the haploid A and B subtypes, according to its haploid configuration. Haploid A contains frame gene KIR2DL1, KIR2DL3, KIR3DL1 suppressor gene, and KIR2DS4 activating gene. Other configurations are collectively referred to as haploid B. Haploid B contains a number of activating genes.

HLA-Cw is distributed widely in human tissue cells, specifically recognized by KIR and involved in the regulation of NK 
cell function, which is the basis of NK cell recognition of self and non-self molecules. When the same HLA-Cw molecule is combined with different KIRs, it produces different signals to activate or inhibit the killing function of the NK cells (8). Observations have revealed that there are differences between individuals in the combined forms of HLA-Cw and SKIR or iKIR, which not only affect susceptibility to disease (9) but also disease progression (10). In order to understand the method of the recognition of HLA-Cw and KIR in a Chinese population, the KIR and its HLA-Cw ligands of 154 normal Han individuals from Jilin were examined by PCR-SSP and the identification of HLA-Cw and KIR was analyzed, to provide a the theoretical basis for future research concerning its role in disease progression.

\section{Materials and methods}

Objective of the study. A total of 154 normal specimens were selected from unpaid blood donors from a Jilin Han population. This group contained 84 males and 70 females (no blood relations) aged between 21 and 50 years old. Venous blood $(5 \mathrm{ml})$ was obtained, placed in test tubes containing EDTA anticoagulants and stored in a $-80^{\circ} \mathrm{C}$ refrigerator. The experimental protocol was established according to the guidelines of the Declaration of Helsinki and was approved by Human Ethics Committee of Jilin University. Informed consent was obtained from each subject prior to their inclusion in the study.

Genomic DNA extraction. Using the TIANamp Blood DNA kit (Tiangen Biotech Beijing Co. Ltd., Beijing, China), genomic DNA was extracted according to the manufacturer's instructions. The DNA concentration was $\geq 80 \mathrm{mg} / \mathrm{l}$ and the purity was $\mathrm{A}_{260} /_{280}=1.65-1.90$.

Primer synthesis. A previously published method for synthesizing the amplified KIR genes using PCR-SSP genotyping primers (Table I) was used (11). The internal control primers of reactions 1 to 15 were FGH (5'-GCCTTCCCAACC ATTCCCTTA-3') and RGH (5'-TCACGGATTTCTGTT GTGTTTC-3'). The length of the amplification product was $429 \mathrm{bp}$; the internal contrast primers of reaction 16 were DRB1-F (5'-TGCCAAGTGGAGCACCCAA-3') and DRB1-R (5'-GCATCTTGCTCTGTGCAGAT-3') and the length of the amplification products was $796 \mathrm{bp}$. The primers of the HLA-Cw gene for PCR-SSP genotyping were in accordance with those of Mandelboim et al (5). The primers are shown in Table II. All primers were synthesized by Beijing DingGuo ChangSheng Biotechnology Co., Ltd. (Beijing, China) and verified by BLAST.

KIR genes and the HLA-Cw PCR-SSP classification. Each KIR gene required two pairs of KIR gene primers (upstream and downstream) plus an internal contrast primer system. The HLA-Cw gene also required upstream and downstream primers and an internal contrast primer system.

To amplify KIR genes by PCR-SSP (12), $1 \mu \mathrm{l}$ of each primer $\operatorname{mix}(5 \mu \mathrm{M})$ was dispensed into separate wells in 384-well plates. Each sample required two vertical rows of wells but KIR2DS1 and KIR3DP1 required only one well. PCR cocktails were prepared for each sample with a total volume of $132 \mu \mathrm{l}$ (enough for 33 reactions) as follows: $200 \mathrm{ng}$ DNA, $16.5 \mu \mathrm{l} 10 \mathrm{X}$ PCR

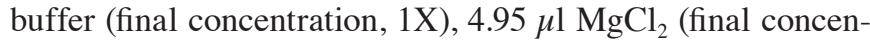
tration $1.5 \mathrm{mM}$ ), $1.32 \mu \mathrm{l} \mathrm{dNTP}$ (final concentration $200 \mathrm{mM}$ ) and $0.825 \mu \mathrm{l} \mathrm{Taq}$ polymerase. PCR cocktail $(4 \mu \mathrm{l})$ was added to each primer mix (total PCR volume $=5 \mu \mathrm{l}$ ). The samples were amplified using a programmable thermal cycler with a heated lid using the following parameters: $3 \mathrm{~min}$ at $94^{\circ} \mathrm{C}, 5$ cycles of $15 \mathrm{sec}$ at $94^{\circ} \mathrm{C}, 15 \mathrm{sec}$ at $65^{\circ} \mathrm{C}$ and $30 \mathrm{sec}$ at $72^{\circ} \mathrm{C} ; 21 \mathrm{cycles}$ of $15 \mathrm{sec}$ at $94^{\circ} \mathrm{C}, 15 \mathrm{sec}$ at $60^{\circ} \mathrm{C}$ and $30 \mathrm{sec}$ at $72^{\circ} \mathrm{C} ; 4$ cycles of $15 \mathrm{sec}$ at $94^{\circ} \mathrm{C}, 1 \mathrm{~min}$ at $55^{\circ} \mathrm{C}$ and $2 \mathrm{~min}$ at $72^{\circ} \mathrm{C}$ with a final 7-min extension step at $72^{\circ} \mathrm{C}$.

The HLA-Cw genes were amplified using PCR-SSP genotyping (12). The PCR system was as follows: In each reaction, $100 \mathrm{ng}$ of genomic DNA was amplified in $15 \mu \mathrm{l}$ of 1X PCR master mix [67 mM Tris- $\mathrm{HCl}, \mathrm{pH} 8.8,16.6 \mathrm{mM}$ $\left(\mathrm{NH}_{4}\right)_{2} \mathrm{SO}_{4}, 0.1 \%$ Tween-20, $\left.2 \mathrm{mM} \mathrm{MgCl} 2,200 \mathrm{mM} \mathrm{dNTP}\right]$ containing specific $(0.1-0.8 \mu \mathrm{m})$ and control primers, as well as 0.5 units $\mathrm{Taq}$ polymerase. PCR conditions were: $2 \mathrm{~min}$ at $94^{\circ} \mathrm{C}, 10$ cycles of $10 \mathrm{sec}$ at $94^{\circ} \mathrm{C}$ and $60 \mathrm{sec}$ at $65^{\circ} \mathrm{C} ; 20$ cycles of $10 \mathrm{sec}$ at $94^{\circ} \mathrm{C}, 50 \mathrm{sec}$ at $61^{\circ} \mathrm{C}$ and $30 \mathrm{sec}$ at $72^{\circ} \mathrm{C}$ with a final 10 -min extension step at $72^{\circ} \mathrm{C}$.

Gel electrophoresis. The amplification products $(5 \mu \mathrm{l})$ were electrophoresed on $2 \%$ agarose gels with a migration distance of $\sim 3 \mathrm{~cm}$ and stained with ethidium bromide. The amplification was evaluated on a UV transilluminator and photographed.

Analysis of the recognition of $H L A-C w$ and KIR. The difference in the method of recognition between various HLA-Cws and KIRs depended on the 80th amino acid residues of the HLA-Cw. If the residue was lysine (Lys; HLA-Cw2 ${ }^{\text {Lys } 80}$ ), the receptor was KIR2DL1/S1. If the residue was asparagine (Asn; HLA-Cw1 ${ }^{\mathrm{Asn} 80}$ ), the receptor was KIR2DL2/L3/S2. The amino acid sequence was acquired according to the HLA-Cw classification results. By combining this information with the phenotype of the KIR, separate HLA-Cw and KIR recognition statistics could be analyzed.

Statistical analysis. The statistical analysis followed that of Martin and Carrington (11) and also included the frequency and genotype frequency of HLA-Cw and KIR in the Han population of Jilin. The KIR gene-frequency $(\mathrm{F})$ was measured by direct counting, the phenotype frequency $(\mathrm{pf} ; \%)=$ total number of positive gene / total nu,ber of research group; the KIR gene frequency was calculated as $F=1-\sqrt{ }(1-p f)$.

\section{Results}

Electrophoresis of the KIR gene. Electrophoretic analysis following the conventional amplification of the KIR gene revealed that the size of the target fragment was consistent with the expected results (Fig. 1).

Electrophoresis of the HLA-Cw gene. Electrophoretic analysis following the conventional amplification of the HLA-Cw gene revealed that the size of the target fragment was consistent with the expected results (Fig. 2).

Phenotype and genotype frequencies of HLA-Cw and KIR in the Han population in Jilin. The results are shown in Table III. 
Table I. Primers used for KIR genotyping by PCR-SSP.

\begin{tabular}{|c|c|c|c|c|c|}
\hline Gene & Primer & Sequence & Primer & Sequence & Size (bp) \\
\hline \multirow[t]{2}{*}{ 2DL1 } & F1 & GTTGGTCAGATGTCATGTTTGAA & R1 & GGTCCCTGCCAGGTCTTGCG & 146 \\
\hline & F2 & TGGACCAAGAGTCTGCAGGA & $\mathrm{R} 2$ & TGTTGTCTCCCTAGAAGACG & 330 \\
\hline \multirow[t]{2}{*}{ 2DL2 } & F1 & CTGGCCCACCCAGGTCG & R1 & GGACCGATGGAGAAGTTGGCT & 173 \\
\hline & F2 & GAGGGGGAGGCCCATGAAT & R2 & TCGAGTTTGACCACTCGTAT & 151 \\
\hline \multirow[t]{2}{*}{ 2DL3 } & F1 & CTTCATCGCTGGTGCTG & R1 & AGGCTCTTGGTCCATTACAA & 550 \\
\hline & F2 & TCCTTCATCGCTGGTGCTG & R2 & GGCAGGAGACAACTTTGGATCA & 800 \\
\hline \multirow[t]{2}{*}{ 2DL4 } & F1 & CAGGACAAGCCCTTCTGC & R1 & CTGGGTGCCGACCACT & 254 \\
\hline & $\mathrm{F} 2$ & ACCTTCGCTTACAGCCCG & R2 & CCTCACCTGTGACAGAAACAG & 288 \\
\hline \multirow[t]{2}{*}{ 2DS2 } & F1 & TTCTGCACAGAGAGGGGAAGTA & R1 & GGGTCACTGGGAGCTGACAA & 175 \\
\hline & F2 & CGGGCCCCACGGTTT & $\mathrm{R} 2$ & GGTCACTCGAGTTTGACCACTCA & 240 \\
\hline \multirow[t]{2}{*}{ 2DS3 } & F1 & TGGCCCACCCAGGTCG & R1 & TGAAAACTGATAGGGGGAGTGAGG & 242 \\
\hline & F2 & CATTGACATGTACCATCTATCCAC & $\mathrm{R} 2$ & AAGCAGTGGGTCACTTGAC & 190 \\
\hline \multirow[t]{2}{*}{ 2DS5 } & $\mathrm{F} 1$ & TGATGGGGTCTCCAAGGG & R1 & TCCAGAGGGTCACTGGGC & 126 \\
\hline & $\mathrm{F} 2$ & ACAGAGAGGGGACGTTTAACC & $\mathrm{R} 2$ & ATGTCCAGAGGGTCACTGGG & 178 \\
\hline \multirow[t]{2}{*}{ 3DL1 } & $\mathrm{F} 1$ & CGCTGTGGTGCCTCGA & R1 & GGTGTGAACCCCGACATG & 191 \\
\hline & F2 & CCCTGGTGAAATCAGGAGAGAG & R2 & TGTAGGTCCCTGCAAGGGCAA & 186 \\
\hline \multirow[t]{2}{*}{ 3DL2 } & F1 & CAAACCCTTCCTGTCTGCCC & R1 & GTGCCGACCACCCAGTGA & 211 \\
\hline & $\mathrm{F} 2$ & CCCATGAACGTAGGCTCCG & $\mathrm{R} 2$ & CACACGCAGGGCAGGG & 130 \\
\hline \multirow[t]{2}{*}{ 3DS1 } & F1 & AGCCTGCAGGGAACAGAAG & R1 & GCCTGACTGTGGTGCTCG & 300 \\
\hline & $\mathrm{F} 2$ & CCTGGTGAAATCAGGAGAGAG & R2 & GTCCCTGCAAGGGCAC & 180 \\
\hline \multirow[t]{2}{*}{ 3DL3 } & F1 & GTCAGGACAAGCCСТTCCTC & R1 & GAGTGTGGGTGTGAACTGCA & 232 \\
\hline & $\mathrm{F} 2$ & TTCTGCACAGAGAGGGGATCA & R2 & GAGCCGACAACTCATAGGGTA & 165 \\
\hline \multirow[t]{2}{*}{ 2DL5 } & F1 & GCGCTGTGGTGCCTCG & R1 & GACCACTCAATGGGGGAGC & 214 \\
\hline & $\mathrm{F} 2$ & TGCAGCTCCAGGAGCTCA & R2 & GGGTCTGACCACTCATAGGGT & 191 \\
\hline \multirow[t]{2}{*}{ 2DP1 } & $\mathrm{F} 1$ & GTCTGCCTGGCCCAGCT & R1 & GTGTGAACCCCGACATCTGTAC & 205 \\
\hline & F2 & CCATCGGTCCCATGATGG & $\mathrm{R} 2$ & CACTGGGAGCTGACAACTGATG & 89 \\
\hline \multirow[t]{2}{*}{ 2DS1 } & $\mathrm{F} 1$ & CTTCTCCATCAGTCGCATGAA & R1 & AGAGGGTCACTGGGAGCTGAC & 102 \\
\hline & $\mathrm{F} 2$ & CTTCTCCATCAGTCGCATGAG & & & \\
\hline \multirow[t]{2}{*}{ 2DS4 } & F1 & CTGGCCCTCCCAGGTCA & R1 & TCTGTAGGTTCCTGCAAGGACAG & 204 \\
\hline & $\mathrm{F} 2$ & GTTCAGGCAGGAGAGAAT & $\mathrm{R} 2$ & GTTTGACCACTCGTAGGGAGC & $197 / 219$ \\
\hline \multirow[t]{2}{*}{ 3DP1 } & F1 & GGTGTGGTAGGAGCCTTAG & R1 & GAAAACGGTGTTTCGGAATAC & 280 \\
\hline & $\mathrm{F} 2$ & CGTCACCCTCCCATGATGTA & & & 395 \\
\hline
\end{tabular}

KIR, killer cell immunoglobulin-like receptor; F1/F2, forward primers; R1/R2, reverse primers.

Table II. Primers used for HLA-Cw genotyping by PCR-SSP.

\begin{tabular}{|c|c|c|c|c|c|}
\hline \multirow[b]{2}{*}{ Gene } & \multicolumn{2}{|r|}{ Forward primers } & \multicolumn{2}{|r|}{ Reverse primers } & \multirow[b]{2}{*}{ Size $(b p$} \\
\hline & Primer & Sequence & Primer & Sequence & \\
\hline HLA-C1 ${ }^{\text {Asn80 }}$ & $\mathrm{F}$ & GAGGTGCCCGCCCGGCGA & $\mathrm{R}$ & CGCGCAGGTTCCGCAGGC & 332 \\
\hline HLA-C2 ${ }^{\text {Lys80 }}$ & $\mathrm{F}$ & GAGGTGCCCGCCCGGCGA & $\mathrm{R}$ & CGCGCAGTTTCCGCAGGT & 332 \\
\hline
\end{tabular}

Recognition of HLA-C2 $2^{\text {Lys }}$ and KIR. The HLA-C2 $2^{\text {Lys }}$ expression frequency was $27.92 \%$, while 72.08 and $21.43 \%$ of individuals expressed only KIR2DL1 or KIR2DS1, respectively, without HLA-C2 ${ }^{\text {Lys }}$. Table IV shows the distribution 
Table III. Phenotype and genotype frequency of KIR and HLA-Cw in the Jilin Han population.

\begin{tabular}{|c|c|c|c|}
\hline Gene & Number & Phenotype frequency $(\%)$ & Genotype frequency \\
\hline 2DL1 & 153 & 99.351 & 0.919 \\
\hline 2DL2 & 21 & 13.636 & 0.071 \\
\hline 2DL3 & 153 & 99.351 & 0.919 \\
\hline 2DL4 & 154 & 100 & 1 \\
\hline 2DL5 & 49 & 31.818 & 0.174 \\
\hline 3DL1 & 143 & 92.857 & 0.733 \\
\hline 3DL2 & 154 & 100 & 1 \\
\hline 3DL3 & 154 & 100 & 1 \\
\hline 2DS2 & 19 & 12.338 & 0.064 \\
\hline 2DS3 & 18 & 11.688 & 0.06 \\
\hline 2DS5 & 37 & 24.026 & 0.128 \\
\hline 3DS1 & 43 & 27.922 & 0.151 \\
\hline 2DP1 & 153 & 99.351 & 0.919 \\
\hline 2DS1 & 48 & 31.169 & 0.17 \\
\hline $3 \mathrm{DP} 1 * 003$ & 76 & 49.351 & 0.288 \\
\hline 3DP1*001/002/004 & 153 & 99.351 & 0.919 \\
\hline 2DS4*001/002 & 113 & 73.377 & 0.484 \\
\hline 2DS4*003-007 & 72 & 46.753 & 0.27 \\
\hline HLA-C1 ${ }^{\text {Asn80 }}$ & 123 & 79.87 & 0.551 \\
\hline HLA-C2 $2^{\text {Lys80 }}$ & 43 & 27.922 & 0.151 \\
\hline
\end{tabular}

KIR, killer cell immunoglobulin-like receptor; HLA-Cw, human leukocyte antigen-Cw; Asn, asparagine; Lys, lysine.

Table IV. The combined frequencies of various compound KIR-HLA genetypes in the Jilin Han population.

\begin{tabular}{lccc}
\hline iKIR+HLA & Frequency $(\%)$ & sKIR+HLA & Frequency $(\%)$ \\
\hline HLA-C1 ${ }^{\text {Asn80 }}+2$ DL2/2DL3 & 68.83 & 2DS2+HLA-C1 ${ }^{\text {Asn80 }}$ & 9.74 \\
HLA-C2 ${ }^{\text {Lys80 }+2 D L 1 ~}$ & 27.27 & 2DS1+HLA-C2 & \\
\hline
\end{tabular}

KIR, killer cell immunoglobulin-like receptor; iKIR, inhibitory KIR; sKir, stimulatory KIR; HLA, human leukocyte antigen; Asn, asparagine; Lys, lysine.

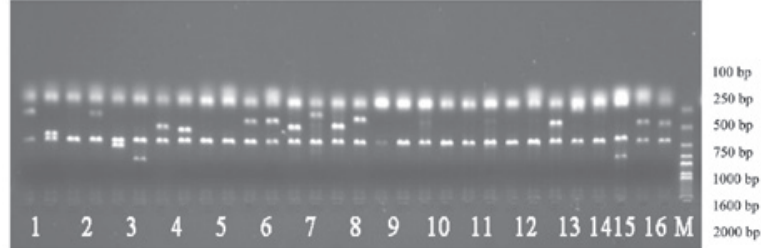

Figure 1. KIR genotyping by PCR-SSP. Lanes 1-16, the PCR-SSP products of KIR2DL1, KIR2DL2, KIR2DL3, KIR2DL4, KIR2DL5, KIR3DL1, KIR3DL2, KIR3DL3, KIR2DS2, KIR2DS3, KIR2DS5, KIR3DS1, KIR2DP1, KIR2DS1 and KIR3DP1, respectively; M, Marker 2000; KIR, killer cell immunoglobulin-like receptor.

of the HLA-Cw KIR activating and inhibitory pairing in the Han population of Jilin. The frequency of HLA-C2 ${ }^{\mathrm{Ly} y 80}+2 \mathrm{DL} 1$ was $27.27 \%$ and that of 2DS1+HLA-C2 $2^{\mathrm{Ly} y 80}$ was $9.74 \%$. Of the studied individuals, $21.43 \%$ expressed only KIR2DS1 without HLA-C2 ${ }^{\text {Lys }}$-KIR2DL1.

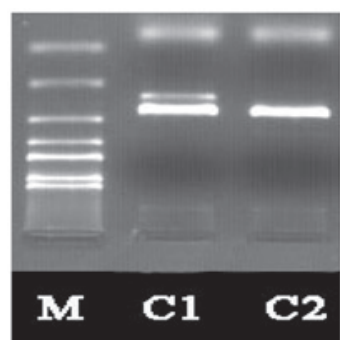

Figure 2. HLA-Cw genotyping by PCR-SSP. M, Marker 2000; C1, PCR-SSP products of HLA-Cw1 ${ }^{\text {Asn }}$; C2, PCR-SSP products of HLA-Cw2 ${ }^{\text {Lys }}$; HLA-Cw, human leukocyte antigen- $\mathrm{Cw}$.

Recognition of HLA-C $1^{\text {Asn }}$ KIR. The HLA-C1 ${ }^{\text {Asn80 }}$ expression frequency was $79.87 \%$, notably higher than the expression frequency of HLA-C $2^{\mathrm{Ly} y 80}$ which was $27.92 \%$, while $34.42 \%$ of individuals expressed only HLA-C1 ${ }^{\text {Asn }}$ without $\mathrm{iKIR}$ or sKIR. Table IV shows the distribution of the HLA-Cw KIR activating 
and inhibitory pairings in the Han population of Jilin. The frequency of HLA-C1 ${ }^{\text {Asn80 }}+2 \mathrm{DL} 2 / 2 \mathrm{DL} 3$ was $68.83 \%$ and that of 2DS2+HLA-C1 ${ }^{\mathrm{Asn} 80}$ was $9.74 \%$. Of the studied individuals, $2.60 \%$ expressed only KIR2DS2 without HLA-C1 ${ }^{\text {Asn }}$-KIR2DL2/L3.

\section{Discussion}

NK cells recognize virus-infected tissue and tumor cells, then modulate the immune response by killing the target cells through cytotoxic effects or secreting cytokines. NK cells constitute the first barrier of the human immune system $(13,14)$. Immature NK cells must be 'educated' by the MHC HLA-Class I molecules, in order to become mature NK cells (15). NK cells adjust their functional status precisely through inhibiting or activating receptors on the membrane surface. In the long-term coevolution of the KIR and the HLA, now highly efficient and accurate receptorligand system is formed gradually. (16). Previously, in a study of 477 cases of malaria, Hirayasu et al (17) observed that there was a marked correlation between the KIR2DL3 gene and its ligand HLA-C1 in the cerebral malaria patients compared with non-cerebral malaria patients. Further research revealed that the frequency of the combination of the KIR2DL3-HLA-C1 in the malaria high-risk population was maintained at a low level.

Thus, the KIR and HLA combination system is important in regulating NK cell function. Therefore, determining the method of recognition of the HLA-Cw and KIR is particularly important. A total of 154 Han individuals from Jilin were selected. The KIR and HLA-Cw genotyping results revealed that the expression frequency of HLA-C1 ${ }^{\mathrm{Asn} 80}$ was $79.87 \%$, lower than the $97.5 \%$ of a Han population in Guangdong (18) and the $76 \%$ of a population in Iran (12). The expression frequency of HLA-C2 $2^{\text {Lys } 80}$ in the Han population of Jilin was $27.92 \%$, which was equal to that of the Guangdong Han population (18) and lower than the frequency in the Iranian population (12). The frequency of HLA-C1 ${ }^{\mathrm{Asn} 80}$ in the Guangdong Han population was $97.5 \%$, which was significantly higher than that of the Jilin Han population. Compared with other populations, the combination frequency of the KIR receptor and its ligand in the Jilin Han population was essentially the same. The frequency of KIR2DS2+HLA-C1 ${ }^{\text {Asn80 }}$ in the Han population of Jilin was $9.74 \%$, less than that of the Guangdong Han (14\%) and Iranian (21.5\%) populations. The Jilin Han HLA-C1 ${ }^{\text {Asn80 }}+\mathrm{KIR} 2 \mathrm{DL} 2 / 2 \mathrm{DL} 3$ frequency was $68.83 \%$, higher than in the Guangdong Han (41.8\%) and Iranian (6.5\%) populations. These observations may be due to ethnic and regional differences of the HLA and KIR.

In the 154 Han individuals from Jilin, it was also observed that $9.74 \%$ of individuals expressed only the combination of 2DS2+HLA-C1 ${ }^{\text {Asn80 }}$ and $9.74 \%$ expressed only the combination of 2DS1+HLA-C2 ${ }^{\mathrm{Ly} y 80}$. For these individuals, due to the inhibitory signal being unable to completely block the activation of signal transduction, their NK cells may attack other tissues, resulting in autoimmune diseases (10). However, this has yet to be confirmed in patients through larger sample and clinical experiments.

Martin et al (9) suggested that the ligands of sKIRs may not be HLA antigens, but bacterial proteins. The authors observed that the KIR2DL1 and KIR2DL2/L3 frequency was high, up to $100 \%$. Therefore the individual expression of HLA-Cw indicates that a corresponding inhibitory ligand receptor is activated. NK cells with sKIR lacking a corresponding HLA-Cw (for example, KIR2DS1 without HLA-Cw2 ${ }^{\text {Lys }}$ ) may be activated by bacterial proteins, resulting in autoimmune diseases. In the current study, $21.43 \%$ of individuals were observed to express KIR2DS1 without HLA-Cw2 ${ }^{\text {Lys }}$ inhibitory receptor ligands. Due to the expression frequency of HLA-Cw1 ${ }^{\text {Asn }}$ being up to $79.87 \%$, no expression of KIR2DS2 only was observed. However, $2.60 \%$ of the population expressed KIR2DS2 but not HLA-Cw1 ${ }^{\mathrm{Asn}}$ inhibitory receptor ligands. The susceptibility to autoimmune disease of such individuals is worthy of investigation.

\section{Acknowledgements}

This study was supported by grants from the National Natural Science Foundation of China (No. 30972610). The authors would like to thank Medjaden Bioscience Limited for assisting in the preparation of this manuscript.

\section{References}

1. Parham P: Immunogenetics of killer cell immunoglobulin-like receptors. Mol Immunol 42: 459-462, 2005.

2. Guethlein LA, Abi-Rached L, Hammond JA and Param P: The expanded cattle KIR genes are orthologous to the conserved single-copy KIR3DX1 gene of primates. Immunogenetics 59: 517-522, 2007.

3. van Bergen J, Thompson A, Retière $\mathrm{C}$, et al: Cutting edge: killer Ig-like receptors mediate 'missing self' recognition in vivo. J Immunol 182: 2569-2572, 2009.

4. Lanier LL: Missing self, NK cells, and The White Album. J Immunol 174: 6565, 2005.

5. Mandelboim O, Reyburn HT, Valés-Goméz M, et al: Protection from lysis by natural killer cells of group 1 and 2 specificity is mediated by residue 80 in human histocompatibility leukocyte antigen $\mathrm{C}$ alleles and also occurs with empty major histocompatibility complex molecules. J Exp Med 184: 913-922, 1996.

6. Moesta AK, Norman PJ, Yawata M, et al: Synergistic polymorphism at two positions distal to the ligand-binding site makes KIR2DL2 a stronger receptor for HLA-C than KIR2DL3. J Immunol 180: 3969-3979, 2008.

7. Stern M, Ruggeri L, Capanni M, et al: Human leukocyte antigens A23, A24, and A32 but not A25 are ligands for KIR3DL1. Blood 112: 708-710, 2008.

8. Xiaolin Yin: The evolution, expression and function of KIR gene. Immunology 20: S103-S105, 2004.

9. Martin MP, Nelson G, Lee JH, et al: Cutting edge: susceptibility to psoriatic arthritis: influence of activating killer Ig-like receptor genes in the absence of specific HLA-Cw alleles. J Immunol 169: 2818-2822, 2002.

10. Yen JH, Moore BE, Nakajima T, et al: Major histocompatibility complex class I-recognizing receptors are disease risk genes in rheumatoid arthritis. J Exp Med 193: 1159-1167, 2001.

11. Martin MP and Carrington M: KIR locus polymorphisms: genotyping and disease association analysis. Methods Mol Biol 415: 49-64, 2008.

12. Tajik N, Shahsavar F Nasiri M and Radjabzadeh MF: Compound KIR-HLA genotype analyses in the Iranian population by a novel PCR-SSP assay. Int J Immunogenet 37: 159-168, 2010.

13. Wodnar-Filipowicz A and Kalberer CP: Function of natural killer cells in immune defence against human leukaemia. Swiss Med Wkly 137 (Suppl 155): 25S-30S, 2007.

14. Cheent $\mathrm{K}$ and Khakoo SI: Natural killer cells: integrating diversity with function. Immunology 126: 449-457, 2009.

15. Brodin P, Kärre K and Höglund P: NK cell education: not an on-off switch but a tunable rheostat. Trends Immunol 30: 143-149, 2009.

16. Single RM, Martin MP, Gao X, et al: Global diversity and evidence for coevolution of KIR and HLA. Nat Genet 39: 1114-1119, 2007.

17. Hirayasu K, Ohashi J, Kashiwase K, et al: Significant association of KIR2DL3-HLA-C1 combination with cerebral malaria and implications for co-evolution of KIR and HLA. PLoS Pathog 8: e1002565, 2012.

18. Xiaolin Yin, Lulu Xiao and Kunyuan Guo: The analysis of HLA-Cw and KIR in 79 Han people of Guangdong. China Immunology 21: 605-607, 2005. 